\title{
Ethanol-Induced Alterations in Electroencephalographic Activity in Adult Males
}

\author{
Howard L. Cohen, Ph.D., Bernice Porjesz, and Henri Begleiter
}

The present investigation examined the effects of placebo (P), low-dose (LD), and high-dose (HD) ethanol on electroencephalographic (EEG) activity in 21 healthy, adult males $(\bar{X}=22.7$ years). Only one condition $(P$, $L D$, or $H D$ ) was presented per day and the condition order was randomized. For each subject, blood-alcohol levels measured via breathalyzer and EEG activity, using the entire 10/20 International System, were recorded both prior to and at intervals of 35, 70, 105, and 140 minutes after $P, L D$, or HD administration. The Fast Fourier Transform was used to calculate power spectral densities for each EEG recording. Measures of the relative areas under the power spectral curve were made for each of the following frequency bands: slow alpha (7.5 to $10 \mathrm{~Hz})$; fast alpha (10.5 to $13.0 \mathrm{~Hz})$; slow beta $(13.5$ to $19.5 \mathrm{~Hz})$; and fast beta (20 to $26 \mathrm{~Hz}$ ) at electrodes F3, F4, C3, C4, P3, P4, O1, and O2. Repeated-measures multivariate analysis of variance performed on normalized relative area values revealed that ethanol had significant effects on $E E G$ activity at anterior sites: frontal $(F 3, F 4)$ and central $(C 3, C 4)$ that presented as increased activity in the slow alpha frequency band. These results suggest a differential responsivity of both cortical region and EEG frequency band to the effects of ethanol ingestion. [Neuropsychopharmacology 8:365-370, 1993]
KEY WORDS: Alpha; Beta; EEG; Power Spectra; Ethanol Investigations into the effects of acute ethanol administration on adult, human electroencephalographic (EEG) activity have reported both decreases in the predominant baseline frequency and increases in power spectral energy (Davis et al. 1941; Engel and Rosenbaum, 1945; Holmberg and Martens, 1955; Begleiter and Platz 1972; Lehtinen et al. 1981; Lukas et al. 1986; Michel and Battig 1989). However, defining the relationship between ethanol ingestion and its effect on the EEG is complicated by numerous variables whose influence

From the Neurodynamics Laboratory, Department of Psychiatry, State University of New York Health Science Center Brooklyn, Brooklyn, New York.

Address correspondence to: Dr. Howard L. Cohen, Neurodynamics Laboratory, Department of Psychiatry, Box 1203, S.U.N.Y. Health Science Center Brooklyn, 450 Clarkson Avenue, Brooklyn, New York 11203.

Received August 14, 1992; revised December 1, 1992; accepted December 8, 1992. must be carefully determined. These include 1) electrode locations analyzed, 2) EEG frequency band(s) examined, 3) ethanol concentration ingested, 4) measure(s) of EEG activity used, 5) predrug EEG activity level, 6) time course over which EEG changes are examined, 7) individual's drinking history, and 8) family history of alcoholism. Even after controlling for these variables, both EEG and subjective responses to acute ethanol ingestion may be highly variable across individuals and within the same individual on different occasions (Begleiter and Platz 1972; Lukas et al. 1986).

The majority of studies have focused on the alpha frequency band, although investigators have not always agreed upon its range or its composition. For example, although some have defined alpha as a continuous band (Holmberg and Martens 1955; Lukas et al., 1986; Kaplan et al. 1988), others have divided alpha into both a slow band and a fast band (Pollock et al. 1983; Volavka et al. 1985; Ehlers and Schuckit 1988, 1991; Ehlers et al. 1989; Lukas et al. 1989). In general, those studies that 
defined alpha as a continuous band reported that ethanol ingestion increased alpha energy. In contrast, the dichotomized band studies have typically reported increased slow alpha energy and either no change (Ehlers and Schuckit 1988, 1991), a decrease (Ehlers et al. 1989), or both increases and decreases (Pollock et al. 1983; Volavka et al. 1985; Lukas et al. 1989) in fast alpha energy.

The changes in alpha energy following ethanol ingestion are frequently, but not always, accompanied by a decrease in the predominant alpha frequency (Davis et al. 1941; Engel and Rosenbaum 1945; Lehtinen et al. 1981; Pollock et al. 1983; Lukas et al. 1986) and, as in the studies of changes in alpha energy, dichotomized alpha band studies report differential effects (Ehlers et al. 1989). Additionally, there is evidence that both the probability and the magnitude of the decrease may be influenced by the individual's baseline EEG activity (Engel and Rosenbaum 1945; Begleiter and Platz 1972; Lukas et al. 1986).

Reports of ethanol-induced changes in faster activity (beta) have also been contradictory. There have been observations of decreased beta power with subsequent replacement by alpha activity (Engel and Rosenbaum 1945; Murphree et al. 1970; Ehlers et al. 1989), increased beta activity (Holmberg and Martens 1955; Murphree et al. 1970), both increases and decreases in mean frequency in the beta range (Lehtinen et al. 1978), and no ethanol-induced effects on beta (Lolli et al. 1964; Ehlers and Schuckit 1988, 1990; Lukas et al. 1989). Additionally, there is evidence (Ehlers et al. 1989; Ehlers and Schuckit 1990) that ethanol-induced changes in beta activity may be influenced by an individual's drinking history. "Moderate" drinkers, in contrast to "low" drinkers, had more beta energy and a higher peak frequency both at baseline and at 90 minutes postethanol ingestion.

The rise and fall in blood-alcohol level following ethanol ingestion is influenced by factors such as the volume and concentration of alcohol ingested and the time period over which ingestion occurs. Attempts to correlate the temporal pattern of EEG changes with the dynamic phases of the blood-alcohol curve have yielded inconsistent results. For example, there are reports that during the ascending phase, increases in alpha energy were greater than during the descending phase (Begleiter and Platz 1972; Lukas et al. 1986), maximal alpha activity coincided with peak blood-alcohol levels (Kaplan et al. 1988), and slowing of the predominant frequency was generaily proportional to the bloodalcohol level (Newman 1959). In contrast, there are observations that EEG changes were greater when blood- and breath-alcohol levels were falling than at corresponding concentrations when the alcohol concentration was rising (Lehtinen et al. 1981), that decreased frequency and increased poweroccurred only after peak blood-alcohol levels were attained, and continued even after these levels had dropped considerably (Davis et al. 1941; Holmberg and Martens, 1955), or that no con. sistent correlations were detected between blood. alcohol concentration and any measure of EEG activity (Ehlers et al. 1989). Lastly, Lehtinen et al. (1981) pointed out that an important factor in the relationship between blood-alcohol level and changes in EEG activity is whether the blood-alcohol level was measured directly from the blood or indirectly from breathalyzer readings. Their study measured both values, but found that when breathalyzer blood-alcohol values were kept constant by upward and downward adjustments of an intravenous ethanol infusion, EEG changes followed directly measured blood-alcohol levels.

Many previous studies of the effects of ethanol on human adult EEG have typically focused on a limited portion of the frequency spectrum, have sampled EEG activity at a small number of electrodes, and have often yielded inconsistent results. It is the intent of the present investigation to sample ethanol-induced changes in EEG activity in both the alpha and beta frequency bands over a larger number of cortical regions than have typically been examined, in a carefully defined population of healthy, adult males. Additionally, most previous investigations have typically used a placebo and a single ethanol dose. In contrast, the subjects in this investigation received placebo, low-dose, and highdose ethanol in a randomized order using a repeated measures design.

\section{METHODS}

\section{Subjects}

The participants in this study were 21 males ranging from 19 to 39 years of age (mean 22.7 years). These individuals had responded to either newspaper ads or to notices for subjects posted in the Health Science Center. An initial screening procedure required the prospec tive subject to fill out a questionnaire detailing his medical and psychiatric history, his drug and alcohol use and those of his relatives. A candidate for the study was rejected if he had any first or second degree relative who was alcoholic. Additional criteria for exclusion included major medical problems, use of medication that affected the central nervous system, or a history of psychiatric problems and/or drug or alcohol abuse. Furthermore, a potential subject was excluded if he had a first or second degree relative with drug abuse or psychiatric problems. Table 1 summarizes the demographic data for these subjects. It includes a "Drink Index," which was obtained by multiplying the number of days per month that the subject drank by the number of drinks per 06 casion. Eighteen of the subjects had a "Drink Index" of less than 20, indicating that the subjects comprised a homogeneous population of light drinkers. 
Table 1. Summary of 21 Subjects' Characteristics

\begin{tabular}{lccc}
\hline & Mean & SD & Range \\
\hline Age (yrs) & 22.7 & 4.51 & $19-39$ \\
$\begin{array}{l}\text { Education (yrs) } \\
\begin{array}{l}\text { Drinking days } \\
\text { per month }\end{array}\end{array}$ & 15.8 & 2.43 & $12-20$ \\
$\begin{array}{l}\text { Drinks per } \\
\text { occasion }\end{array}$ & 4.05 & 3.32 & $1-14$ \\
Drink Index & 2.38 & 1.20 & $1-5$ \\
\hline
\end{tabular}

* The Drink Index is the product of drinking days per month and drinks per occasion.

\section{Experimental Design}

Each subject was tested once under each of three conditions: placebo $(P)$, low-dose (LD) ethanol, or highdose (HD) ethanol. Condition order was randomized and there was a minimum 1-day interval between conditions. In the LD and HD conditions, the subject received a volume (ml) of $100 \%$ ethanol equal to 0.5 and 0.8 times his weight in kilograms, respectively, dissolved in a volume of ginger ale equal to three times that number (see Table 2). In the P condition, the subject drank a volume (ml) of ginger ale equal to twice his weight in kilograms so that the total volume of liquid equalled that of the LD condition. Under each condition the subject ingested the drink over a 10-minute period. A specially designed container (Mendelson et al. 1984) was used to provide an equally strong ethanol odor for both placebo and alcohol conditions. Within the container a small trap held $3 \mathrm{ml}$ of a solution consisting of three parts ethanol dissolved in seven parts ginger ale; this ensured that under each condition, the subject's first taste was ethanol. However, the small amount of ethanol ingested did not produce a measurableblood-alcohol level. Figure 1 presents mean bloodalcohol level $(\mathrm{mg} / 100 \mathrm{ml})$ at four postingestion intervals for both the LD and HD conditions.

Five EEG recordings were made under each of three conditions; the first, approximately 2 minutes before $\mathrm{P}, \mathrm{LD}$, or $\mathrm{HD}$, and the second through fifth at intervals of $35,70,105$, and 140 minutes afterwards. A breathalyzer (Alco-Sensor III; Intoximeters, Inc., St. Louis, MO) was used to monitor the subject's blood-alcohol level initially upon arrival at the laboratory and then immediately preceding each EEG recording.

Table 2. Fluid Volume (ml) Consumed by Each Subject as a Function of Body Weight $(\mathrm{kg})$ for Each Experimental Condition

\begin{tabular}{lcc}
\hline & Vol. of Ethanol & Vol. of Ginger Ale \\
\hline Placebo (P) & 0 & $2.0 \times \mathrm{kg}$ \\
Low dose (L) & $0.5 \times \mathrm{kg}+$ & $3.0(0.5 \times \mathrm{kg})$ \\
High dose $(\mathrm{H})$ & $0.8 \times \mathrm{kg}+$ & $3.0(0.8 \times \mathrm{kg})$ \\
\hline
\end{tabular}

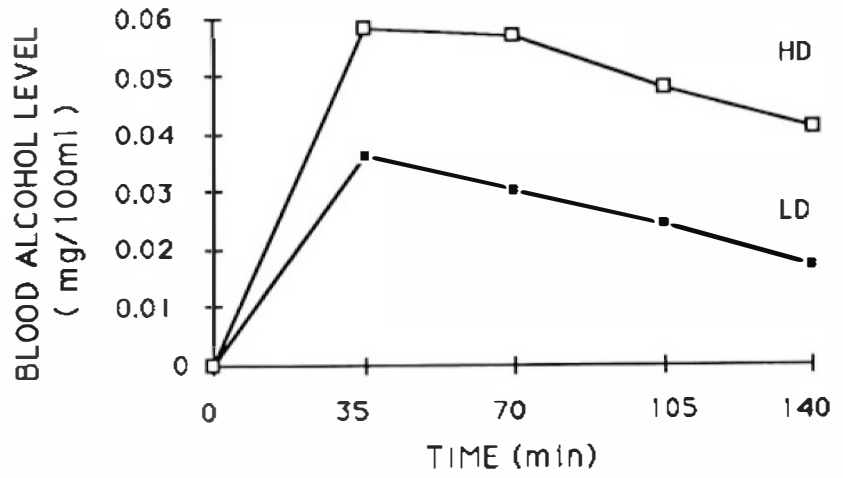

Figure 1. Mean blood-alcohol levels $(\mathrm{mg} / 100 \mathrm{ml})$ as a function of time (minutes) under both the LD (black squares) and HD (white squares) conditions.

\section{Recording Methods and Parameters}

The subject was seated comfortably in a dimly lighted, temperature regulated, sound attenuated chamber (Industrial Acoustics Corp., Bronx, NY). He was told to close his eyes and relax but not to fall asleep. Each subject wore a fitted electrode cap (Electro-Cap International, Inc., Eaton, $\mathrm{OH}$ ) using all 21 electrodes of the $10 / 20$ International System. The nasion served as reference and the forehead as ground. Both vertical and horizontal eye movements were monitored.

Cortical activity was amplified $20 \mathrm{k}$ (bandpass 0.1 to $100 \mathrm{~Hz}$ ) with a Grass Neurodata Acquisition System (Quincy, MA). The data were sampled continuously at $128 \mathrm{~Hz}$ for a period of 120 seconds. Artifact rejection (EMG, EOG, and saturation artifact) was performed blindly, off-line by a highly trained technician.

\section{Data Analysis}

All 21 EEG channels were continuously displayed on a monitor. The first 12 artifact-free, 4-second frames were selected and then analyzed via the Fast Fourier Transform. The consequent power spectral densities were then summed and averaged. This procedure was repeated for each of the five EEG recordings made under the P, LD, and HD conditions. Measures of relative area under the power spectral curve at . $25-\mathrm{Hz}$ intervals were then obtained from the summed and averaged power spectral densities. Determinations of relative area were made for each of the following frequency bands: slow alpha (SA, 7.5 to $10.0 \mathrm{~Hz}$ ); fast alpha (FA, 10.5 to $13.0 \mathrm{~Hz}$ ); slow beta (SB, 13.5 to 19.5 $\mathrm{Hz}$ ); and fast beta (FB, 20 to $26 \mathrm{~Hz}$ ) at electrodes $\mathrm{F} 3$, $\mathrm{F} 4, \mathrm{C} 3, \mathrm{C} 4, \mathrm{P} 3, \mathrm{P} 4, \mathrm{O} 1$, and O2. These measures were then normalized. Statistical analyses consisted of repeated-measures multivariate analysis of variance with Greenhouse-Geisser corrections conducted on normalized, relative area values for each frequency band at each electrode under the P, LD, and HD condition. 
Figure 2. Percent change in normalized, relative SA area (a.u.) (7.5 to 10.0 $\mathrm{Hz}$ ) at each electrode as a function of time (minutes) following $\mathrm{P}$ (black rectangle), LD (white rectangle), and HD (black diamond) ethanol ingestion. The EEG activity was amplified $(20 \mathrm{k})$ and recorded over a bandpass of 0.1 to $100 \mathrm{~Hz}$ with a sampling rate of $128 \mathrm{~Hz}$.

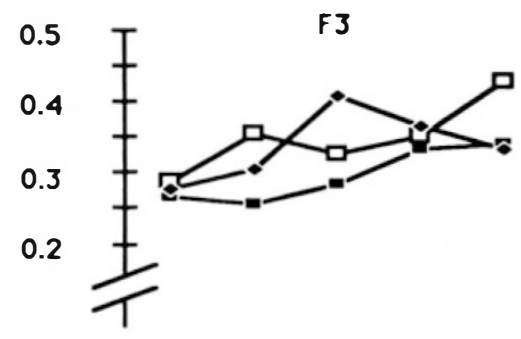

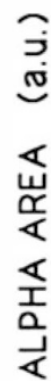

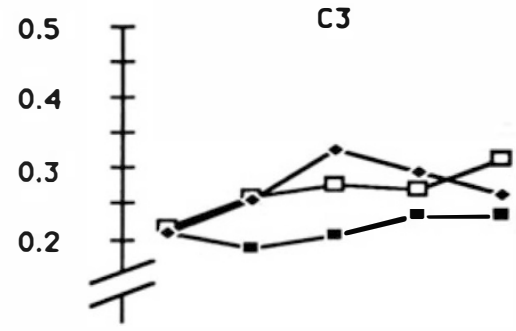

P3
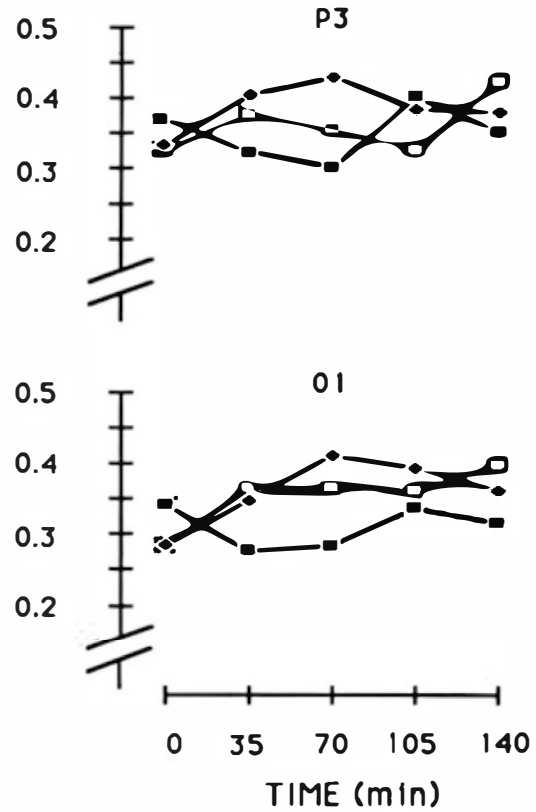

F4

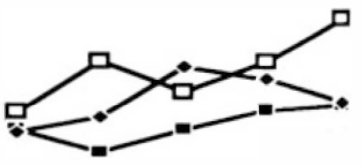

C4

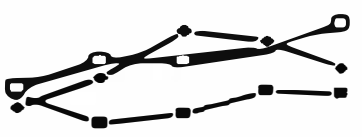

P4

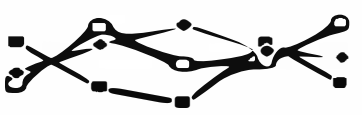

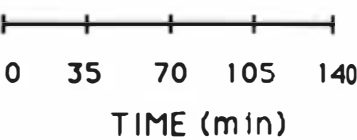

\section{RESULTS}

The results of this study indicate that ethanol ingestion by healthy, adult males induces specific EEG changes manifested as increased activity in the SA (7.5 to 10.0 $\mathrm{Hz}$ ) frequency band. Initially, comparisons of EEG activity were made among electrodes $\mathrm{O} 1, \mathrm{P} 3, \mathrm{C} 3$, and F3, and $\mathrm{O} 2, \mathrm{P} 4, \mathrm{C} 4$, and $\mathrm{F} 4$ to determine whether there were significant response differences across electrodes on the same side. The main effect of "electrode" was significant in both the left hemisphere $(F=[39.68], d f=[3,18]$, $p<.0001)$ and in the right hemisphere $(F=[11.68], d f=$ $[3,18], p<.0002)$. Then, MANOVA was performed on the EEG activity at each electrode, separately. The results of these analyses indicated a significant ethanolrelated increase in SA activity. These increases occurred at frontal electrodes F3 $(F=[4.17], d f=[2,19], p<.03)$,
F4 $(F=[13.58], d f=[2,19], p<.0002), C 3(F=[6.05]$, $d f=[2,19], p<.009)$, and $C 4(F=[4.70], d f=2,19]$, $p<.02)$, but not in the posterior electrodes $\mathrm{P} 3, \mathrm{P} 4$, and $\mathrm{O} 1, \mathrm{O} 2$. Lastly, laterality differences were examined by comparing the activity between electrodes overlying homologous cortical locations. The results indicated significant differences at electrode pairs $\mathrm{C} 3, \mathrm{C} 4(\mathrm{~F}=$ [58.64], $d f=[1,20], p<.0001)$ and $\mathrm{O} 1, \mathrm{O} 2(F=[28.28]$, $d f=[1,20], p<.0001)$. This appears to reflect the fact that at baseline, SA levels at $\mathrm{C} 4$ and $\mathrm{O} 2$ were $24.8 \%$ and $17.2 \%$ higher, respectively, than at $\mathrm{C} 3$ and $\mathrm{O} 1$.

Figure 2 presents, at each electrode, the changes in normalized, relative SA $(7.5$ to $10.0 \mathrm{~Hz})$ activity as a function of time (minutes) after $P, L D$, and $H D$ ethanol ingestion. In general, increases in SA activity occurred after the changes in blood-alcohol level, which tended to peak at between 35 and 70 minutes postin- 
gestion. Elevated SA activity levels were maintained even after blood-alcohol level began to decrease. Although the increases in SA activity did not correspond to changes in blood-alcohol level, a statistically significant dose effect was observed at electrodes F3, F4 and $\mathrm{C} 3, \mathrm{C} 4$, but not at $\mathrm{O} 1, \mathrm{O} 2$ and $\mathrm{P} 3, \mathrm{P} 4$. However, the ethanol-induced increases in SA activity, although significantly greater than those occurring during the $P$ condition, were not significantly different from one another, as demonstrated by the overlying LD and HD functions.

\section{DISCUSSION}

This study demonstrates that ethanol ingestion by healthy, adult males induces an increase in SA activity that occurs at frontal sites (F3, F4 and C3, C4). In contrast, almost no ethanol-induced changes in EEG activity were observed in the posterior locations examined. The ethanol-induced increases in SA activity did not correspond to changing blood-alcohol levels and typically peaked after the blood-alcohol level began to fall. Further, although ethanol produced significantly larger increases in SA activity than did the $P$, there were no differences between the effects of the two doses.

In general, the increase in spectral magnitude in the SA frequency band has been described as the primary EEG response to ethanol ingestion (Davis et al. 1941; Engel and Rosenbaum 1945; Holmberg and Martens 1955; Begleiter and Platz 1972; Lehtinen et al. 1978; 1981; Lukas et al. 1986; Michel and Battig 1989), although some studies reported that the changes in SA were accompanied by altored activity in theta (Lukas etal. 1986; Ehlers et al. 1989) and Fast Alama (Volavka et al. 1985; Lukas et al. 1989, 1990) frequency bands. Additionally, although we recorded significant changes in EEG activity over frontal cortical regions, in contrast, other studies have often reported more widespread ethanol-induced EEG changes (Davis et al. 1941; Holmberg and Marten 1955; Lehtinen et al. 1978, 1981; Volavka et al. 1985; Ehlers and Schuckit 1988; Ehlers et al. 1989; Lukas et al. 1989, 1990).

Regional differences in the electrophysiologic effects of ethanol have also been obtained from studies of event-related potentials (ERP). These studies demonstrated that ethanol both reduced some ERP amplitudes and increased latencies of long latency (>100 msec) components (Salamy and Williams 1973; Porjesz and Begleiter 1975, 1983, 1985); the magnitude of these effects is typically greater in association cortex than in primary sensory cortex (Salamy and Williams 1973; Porjesz and Begleiter 1975, 1983, 1985). It has been suggested that this sensitivity may reflect the complex, polysynaptic structure of association cortex (Himwich and Callison 1972; Kalant 1975). However, cortical neu- roanatomy alone cannot adequately explain our results. For example, although ethanol had a significant effect on SA activity at electrodes F3 and F4, which overlie association cortex in the middle frontal gyrus, it had no effect on SA activity at P3 and P4, which overlie posterior association cortex in the superior parietal lobule (Homan et al. 1987). It is important to recognize that cortical recordings at a specific locus do not necessarily reflect localized activity, but, in fact, may represent afferent volleys originating at distant cortical or subcortical sites. Furthermore, it is not clear that the same mechanisms by which ethanol reduces ERP amplitude and increases component latencies underlies the observed increases in SA activity, which may reflect either excitatory or inhibitory processes. Lastly, it is unlikely that the SA increases, particularly at $\mathrm{C} 3$ and C4, reflected changes in motor activity, as the subjects were relaxed, with eyes closed, and were not required to make any responses.

Although Figures 1 and 2 demonstrate the effects of increased blood-alcohol levels on SA activity at each electrode, significant dose effects were observed only at electrodes F3, F4 and C3, C4. In general, ethanol ingestion significantly increased the magnitude of $\mathrm{SA}$ activity compared with the effects of $P$, although there was no difference between HD and LD as evidenced by the overlapping functions. Interestingly, to our knowledge, there are almost no other studies that have examined the effects of multiple doses of ethanol on human EEG activity in the same subject population. Other studies administering multiple ethanol doses have either used each dose on separate groups of subjects (Lukas et al. 1986), did not record EEG activity under each condition (Ehlers and Schuckit 1988, 1990, 1991), or used different forms of ethanol, such as, bourbon and vodka (Murphree et al. 1970) and red wine and Martini (Lolli et al. 1964). The fact that Figure 2 shows no significant difference between the HD and LD functions suggests that a greater difference between doses may be necessary to elicit a significant dose-dependent difference. However, even a greater difference between ethanol doses may not induce these effects as there is a high degree of inter- and intrasubject variability in both the blood-alcohol and EEG response to ethanol (Lehtinen et al. 1981; Lukas et al. 1986; Porjesz and Begleiter 1983).

Figures 1 and 2 also demonstrate that increases in SA activity lagged behind increases in blood-alcohol level under both the LD and HD conditions, and that increased SA activity typically persisted even after blood-alcohol level decreased. A similar functional relationship, wherein increases in alpha activity peaked and remained elevated during decreasing blood-alcohol level, has been observed by several investigators (Davis et al. 1941; Holmberg and Martens 1955; Lehtinen et al. 1981).

The results of the current investigation indicate that 
the response of the central nervous system to ethanol, as reflected in the analysis of EEG activity, is not uniform. Rather, it appears that ethanol has different local cortical effects and/or may differentially influence subcortical structures whose ascending cortical projections terminate in the cortical loci examined. To investigate these possibilities, our laboratory is currently engaged in ongoing research using both topographic analyses and dipole localization techniques to define sources of EEG activity. Comparisons of ethanol-induced EEG response will then be made between both normal individuals and those at high risk for the development of alcoholism.

\section{ACKNOWLEDGMENTS}

The authors gratefully acknowledge the technical assistance of Peter Allen, Ping-Wu Li, Arthur Stimus, Carrie Tabachnik, and Guowang Zhang.

This work was supported by Grants AA-05524 and AA02686 from NIAAA to $\mathrm{HB}$.

\section{REFERENCES}

Begleiter H, Platz A (1972): The effects of alcohol on the central nervous system. In Kissin B, Begleiter $\mathrm{H}$ (eds), The Biology of Alcoholism, Vol. 2: Physiology and Behavior. New York, Plenum Press, pp 293-343

Davis PA, Gibbs FA, Davis H, Jetter WW, Trowbridge LS (1941): The effects of alcohol upon the electroencephalogram (brain waves). Q J Stud Alcohol 1:626-637

Ehlers CL, Schuckit MA (1988): EEG response to ethanol in sons of alcoholics. Psychopharmacol Bull 24:434-437

Ehlers CL, Schuckit MA (1990): EEG fast frequency activity in the sons of alcoholics. Biol Psychiatry 27:631-641

Ehlers CL, Schuckit MA (1991): Evaluation of EEG alpha activity in sons of alcoholics. Neuropsychopharmacology 4:199-205

Ehlers CL, Wall TL, Schuckit MA (1989): EEG spectral characteristics following ethanol administration in young men. EEG Clin Neurophysiol 73:179-187

Engel GL, Rosenbaum M (1945): Delerium III. Electroencephalographic changes associated with acute alcoholic intoxication. Arch Neurol Psychiatry 53:44-50

Himwich HE, Callison DA (1972): The effect of alcohol on evoked potentials of various parts of the central nervous system of the cat. In Kissin B, Begleiter H (eds), The Biology of Alcoholism. Vol. 2: Physiology and Behavior. New York, Plenum Press, pp 67-84

Holmberg G, Martens S (1955): Electroencephalographic changes in man correlated with blood alcohol concentration and some other conditions following standardized ingestion of alcohol. Q J Stud Alcohol 16:411-424

Homan RW, Herman J, Purdy P (1987): Cerebral location of international 10-20 system electrode placement. EEG Clin Neurophysiol 66:376-382
Kalant H (1975): Direct effects of ethanol on the nervous system. Fed Proc 34:1930-1941

Kaplan RF, Hesselbrock VM, O'Connor S, de Palma N (1988): Behavioral and EEG responses to alcohol in nonalcoholic men with a family history of alcoholism. Prog Neuro. psychopharmacol Biol Psychiatry 12:873-885

Lehtinen I, Lang AH, Keskinen E (1978): Acute effects of small doses of alcohol on the NSD parameters (Normalized Slope Descriptors) of human EEG. Psychopharmacology 60:87-92

Lehtinen I, Lang AH, Jantii V, Pakkanen A, Keskinen E, Nyrke T, Lempiainen M (1981): Ethanol-induced disturbance in human arousal mechanism. Psychopharmacology 73:223-229

Lolli G, Nencini R, Misti R (1964): Effects of two alcoholic beverages on the electroencephalographic and elec. tromyographic tracings of healthy men. Q J Stud Alcohol 25:451-458

Lukas SE, Mendelson JH, Benedikt RA, Jones B (1986): EEC alpha activity increases during transient episodes of ethanol-induced euphoria. Pharmacol Biochem Behav 25:889-895

Lukas SE, Mendelson JH, Woods BT, Mello NK, Teoh SK (1989): Topographic distribution of EEG alpha activity during ethanol-induced intoxication in women. J Stud Alcohol 50:176-185

Lukas SE, Mendelson JH, Kouri E, Bolduc M, Amass L (1990): Ethanol-induced alterations in EEG alpha activity and apparent source of the auditory P300 evoked response potential. Alcohol 7:471-477

Mendelson JH, McGuire M, Mello NK (1984): A new device for administering placebo alcohol. Alcohol 1:417-419

Michel Ch, Battig K (1989): Separate and combined psy. chophysiological effects of cigarette smoking and alco. hol consumption. Psychopharmacology 97:65-73

Murphree HB, Schultz RE, Jusko AG (1970): Effects of high congener intake by human subjects on the EEG. Q J Stud Alcohol Suppl 5:50-61

Newman HW (1959): The effect of alcohol on the electroencephalogram. Stanford Med Bull 17:55-60

Pollock VE, Volavka J, Goodwin DW, Mednick SA, Gabrielli WF, Knop J, Schulsinger F (1983): The EEG after alcohol administration in men at risk for alcoholism. Arch Gen Psychiatry 40:857-861

Porjesz B, Begleiter H (1975): Alcohol and bilaterally evoked brain potentials. Adv Exp Med Biol 59:553-567

Porjesz B, Begleiter H (1983): Brain dysf unction and alcohol. In Kissin B, Begleiter H (eds) The Pathogenesis of Alcoholism, Vol. 7. New York, Plenum Press, pp 415-483

Porjesz B, Begleiter H (1985): Human brain electrophysioogy. In Tarter RE, Thiel DH (eds), Alcohol and the Brain. New York, Plenum Press, pp 139-182

Salamy A, Williams HL (1973): The effects of alcohol on sensory evoked and spontaneous cerebral potentials in man. EEG Clin Neurophysiol 35:3-11

Volavka J, Pollock V, Gabrielli WF Jr, Mednick SA (1985): The EEG in persons at risk for alcoholism. In Galanter M (ed), Recent Developments in Alcoholism. New York, Plenum Press, pp 21-36 